\title{
Quantification of PET Studies
}

\author{
Adriaan A. Lammertsma, $\mathrm{PhD}^{\mathrm{a}}$ \\ a Department of Radiology \& Nuclear Medicine, vU University Medical Center, Amsterdam \\ UMC, Amsterdam, The Netherlands
}

Received Nov 27, 2018; accepted Nov 27, 2018

doi: $10.1007 / \mathrm{s} 12350-018-01583-\mathrm{x}$

\section{See related article, pp. 2034-2044}

Positron emission tomography (PET) is a unique molecular imaging modality, which allows for non-invasive in vivo assessments of regional tissue function in man. ${ }^{1}$ It represents the most selective and sensitive (pico- to nano-molar range) method for measuring molecular pathways and interactions in vivo. ${ }^{2} \mathrm{~A}$ multitude of physiological, biochemical, and pharmacological parameters can be measured, including blood flow (perfusion), blood volume (vascularity), oxygen utilization, glucose metabolism, pre- and post-synaptic receptor density and affinity, neurotransmitter release, enzyme activity, drug delivery and uptake, gene expression, etc. Within cardiology the method has mainly been used for assessing myocardial blood flow (MBF) and glucose metabolism (MMRglu). In fact, the so-called MBF/MMRglu match/mismatch approach ${ }^{3}$ probably was the first diagnostic application of PET and for many years has been the gold standard for assessing myocardial viability. Apart from its use in diagnostic imaging, PET has the capacity to provide new information on human disease, for example by using novel radiotracers. In recent years, PET plays an increasing role in the objective assessment of both progression of disease and therapeutic efficacy. ${ }^{4}$ At present, the latter application has found its way in the development of new drugs, but at the same time there is an enormous potential to use PET as a guiding tool in precision medicine.

Reprint requests: Adriaan A. Lammertsma, $\mathrm{PhD}$, Department of Radiology \& Nuclear Medicine, VU University Medical Center, Amsterdam UMC, P.O. Box 7057, 1007 MB, Amsterdam, The Netherlands; aa.lammertsma@vumc.nl

J Nucl Cardiol 2019;26:2045-7.

1071-3581/\$34.00

Copyright (C) 2019 American Society of Nuclear Cardiology.
For many diagnostic applications visual inspection of reconstructed images is sufficient. One notable exception is, however, the presence of three-vessel disease, where the relative distribution may seem normal and only a quantitative assessment will indicate that there is a global reduction in, for example, $\mathrm{MBF}^{5}$ In addition, recently it has been shown that quantitative MBF measurements have a high accuracy in selecting appropriate patients for catheterisation, much higher than the accuracy of qualitative SPECT assessments. ${ }^{6}$ In addition, quantitative measurements are essential not only for many research applications, but also for longitudinal studies (response assessments), especially in the presence of global effects.

Quantification is not a single-step procedure. On the contrary, many issues need to be addressed when performing quantitative PET studies. These issues are related to data acquisition, data processing, and data analysis (Table 1), although a strict separation is not always possible. For example, in myocardial studies, motion correction usually requires gated acquisitions, i.e., this data processing step can only be carried out by adapting data acquisition accordingly.

In a thorough study published in this issue, ${ }^{7}$ Turco and co-workers combine three issues (image reconstruction, partial volume correction and motion correction) in a single step by defining the reconstruction technique that maximizes quantitative accuracy. To this end, they incorporate partial volume correction in the reconstruction algorithm itself and use gating to correct for respiratory and cardiac motions.

It should be noted that both partial volume and motion corrections are not new. The awareness that the limited resolution of PET scanners may result in partial volume effects (i.e., underestimation of regional tracer concentrations for structures that are smaller than twice the resolution of the scanner) is as old as PET itself, ${ }^{8}$ as are methods developed to correct for these effects. Interestingly, over the years, partial volume effects have become smaller with improvements in spatial resolution (from $\sim 2 \mathrm{~cm}$ in 1980 to $\sim 4 \mathrm{~mm}$ today). Nevertheless, 
Table 1. Quantification issues

Data acquisition issues
Scanner normalization
Injected dose, correcting for activity remaining in the
syringe
Cross-calibration against well-counter (blood) and/or
dose calibrator (injected dose)
Data processing issues
Decay and dead-time corrections
Corrections for random and scattered coincidences
Image reconstruction
Correction for tissue attenuation
Correction for patient motion
Volume of interest definition
Correction for partial volume effects
Data analysis issues
Arterial input function
Tissue time-activity curves
Compartment models
Linearisations, such as the Patlak plot ${ }^{12}$
Semi-quantification, e.g., standardized uptake value
(SuV)

with improving resolution, there is the associated wish to look at smaller structures and substructures (e.g., epiand endocardium separately) and therefore, whenever possible, corrections should be made. Although effects of cardiac and especially respiratory motion have been investigated to a lesser extent than resolution effects, the first study was already published in $1982 .{ }^{9}$ Methods to correct for motion, however, were developed later, as the sensitivity of the first generation of PET scanners did not allow for gating.

Looking at the issues listed in Table 1 it will be clear that the scope of the study by Turco and coworkers is limited. This may seem like a shortcoming of the study, but that certainly is not the case. In fact, all quantification issues should be addressed separately, as each single step needs to be in place before a quantitative PET study can be performed. In other words, quantification fails if any of the components involved in data acquisition, data processing and data analysis fails. As such, the study by Turco and co-workers is an important cog in the wheel.

In the study by Turco and co-workers, partial volume and motion corrections were applied to static $\left[{ }^{18} \mathrm{~F}\right] \mathrm{FDG}$ scans of 30 minutes duration. This is a logical first choice, as it is the most widely used scanning procedure. Nevertheless, the limitations of this approach should also be considered. Firstly, scanning for 30 minutes using the irreversible tracer $\left[{ }^{18} \mathrm{~F}\right] \mathrm{FDG}$ guarantees excellent statistics. In general, partly because of their shorter half-lifes and partly because of reversible kinetics, scans using C-11, N-13 and especially O-15 labeled tracers will be shorter and contain less counts. Consequently, corresponding statistics will be poorer than those of long static $\left[{ }^{18} \mathrm{~F}\right] \mathrm{FDG}$ scans. Clearly, procedures first need to be evaluated under "near-ideal", conditions as done by Turco and co-workers, but further tests are required before these methods can be extrapolated to other conditions (for example, to exclude possible noise induced bias).

The second limitation is more basic. Using a static scan it is only possible to quantify uptake of a tracer, such as $\left[{ }^{18} \mathrm{~F}\right]$ FDG uptake, at a given time. Unless there is equilibrium (rarely the case after a bolus injection), uptake will be time dependent. In addition, net uptake in the myocardium will depend on the total delivery of a tracer through the blood stream and may therefore depend on (changes in) uptake elsewhere in the body. To quantify a physiological or pharmacological process (such as glucose metabolism), dynamic scans (starting at time of injection) are required. From the time course of uptake in tissue (and blood) it is possible to quantify kinetic parameters (independent of time and input) using tracer kinetic modeling. ${ }^{10}$ These dynamic scans consist of many frames (basically a series of successive scans). Immediately after injection, frames need to be very short (as short as 5 seconds) to catch the rapid initial changes in tracer concentration. When these changes become smaller later after injection, frame duration can be increased. Nevertheless, even for $\left[{ }^{18} \mathrm{~F}\right] \mathrm{FDG}$, initial frames will be short and noisy, and the partial volume reconstruction algorithm used by Turco and co-workers needs to be validated under such noisy conditions before the method can be applied to dynamic scans.

For fully quantitative dynamic studies an alternative partial volume correction method is to incorporate partial volume in the tracer kinetic model. The potential of this method has been demonstrated by Iida et al for measuring $\mathrm{MBF}$ using $\left[{ }^{15} \mathrm{O}\right] \mathrm{H}_{2} \mathrm{O} .{ }^{11}$ The principle is simple. Both uptake of freely diffusible water in tissue and its subsequent washout from tissue are flow dependent. As the rate of clearance is independent of partial volume, it can be used to calculate CBF. A comparison with the rate of influx (which is affected by partial volume) then provides a measure of the amount of tissue within the region of interest. Interestingly, an MBF measurement can be added to any tracer study, as it only takes about 5 minutes to acquire the data. In that case, the tissue fraction data can also be used for the other tracer. Note, that this is not an argument against using an independent partial volume correction method within the reconstruction algorithm as, even for $\left[{ }^{15} \mathrm{O}\right] \mathrm{H}_{2} \mathrm{O}$, it is important to make sure that corrections are as small as possible. 
For best results, Turco and co-workers recommend the use of an end-systolic image. This is fine for a long $\left[{ }^{18}\right.$ F]FDG scan, but may be problematic for dynamic scans. Using only one gate from the cardiac cycle means that counts acquired during the other gates are thrown away. As a result image quality of the short dynamic frames may become unacceptable. For dynamic studies it would be best to use all gates, for example by using some form of co-registration between gates.

In summary, quantification of PET studies requires attention to detail, as a series of steps needs to be optimized. Three of those steps, reconstruction algorithm, partial volume correction, and motion correction, have been addressed by Turco and co-workers. They define optimal settings for quantifying (static) $\left[{ }^{18}\right.$ F]FDG uptake in the myocardium. As such, their study is important in those cases where an uptake measurement suffices, e.g., in many diagnostic studies. However, further work is needed before the method can be used in truly quantitative PET studies, i.e., those that attempt to quantify pathophysiological parameters, which require dynamic PET scanning and tracer kinetic modeling.

\section{Disclosure}

Adriaan A. Lammertsma has nothing to disclose.

\section{References}

1. Phelps ME. PET: molecular imaging and its biological applications. Berlin: Springer; 2004.

2. Jones $\mathrm{T}$. The role of positron emission tomography within the spectrum of medical imaging. Eur J Nucl Med. 1996;23:207-11.

3. Tillisch J, Brunken R, Marshall R, Schwaiger M, Mandelkern M, Phelps M, et al. Reversibility of cardiac wall-motion abnormalities predicted by positron tomography. N Engl J Med. 1986;314:8848.

4. Yoshinaga K, Chow BJ, deKemp RA, Thorn S, Ruddy TD, Davies $\mathrm{RA}$, et al. Application of cardiac molecular imaging using positron emission tomography in evaluation of drug and therapeutics for cardiovascular disorders. Curr Pharm Des. 2005;11:903-32.

5. Harms HJ, Huisman MC, Knaapen P, Lammertsma AA, Lubberink M. Parametric imaging of myocardial blood flow and viability using $\left[{ }^{15} \mathrm{O}\right] \mathrm{H}_{2} \mathrm{O}$ and PET/CT. Imaging Med. 2011;3:71124.

6. Danad I, Raijmakers PG, Driessen RS, Leipsic J, Raju R, Naoum $\mathrm{C}$, et al. Comparison of coronary CT angiography, SPECT, PET, and hybrid imaging for diagnosis of ischemic heart disease determined by fractional flow reserve. JAMA Cardiol. 2017;2:1100-7.

7. Turco A, Gheysens O, Duchenne J, Nuyts J, Rega F, Voigt JU, et al. Partial volume and motion correction in cardiac PET: first results from an in vs ex vivo comparison using animal datasets. $\mathrm{J}$ Nucl Cardiol. 2018. https://doi.org/10.1007/s12350-018-01581-z.

8. Hoffman EJ, Huang SC, Phelps ME. Quantitation in positron emission computed tomography: 1 . Effect of object size. J Comput Assist Tomogr. 1979;3:299-308.

9. Ter-Pogossian MM, Bergmann SR, Sobel BE. Influence of cardiac and respiratory motion on tomographic reconstructions of the heart: implications for quantitative nuclear cardiology. J Comput Assist Tomogr. 1982;6:1148-55.

10. Lammertsma AA. Compartmental modeling in emission tomography. In: Grupen C, Buvat I, editors. Handbook of particle detection and imaging. Berlin: Springer; 2012. p. 1065-81.

11. Iida H, Kanno I, Takahashi A, Miura S, Murakami M, Takahashi $\mathrm{K}$, et al. Measurement of absolute myocardial blood flow with $\mathrm{H} 215 \mathrm{O}$ and dynamic positron-emission tomography. Strategy for quantification in relation to the partial-volume effect. Circulation. 1988;78:104-15.

12. Patlak CS, Blasberg RG, Fenstermacher JD. Graphical evaluation of blood-to-brain transfer constants from multiple- time uptake data. J Cereb Blood Flow Metab. 1983;3:1-7.

Publisher's Note Springer Nature remains neutral with regard to jurisdictional claims in published maps and institutional affiliations. 